\title{
Metastasis of ovarian cancer to the breast: A report of two cases and a review of the literature
}

\author{
CLEMENS B. TEMPFER $^{1}$, NARIMAN EL FIZAZI ${ }^{1}$, HASSAN ERGONENC $^{2}$ and WIEBKE SOLASS ${ }^{3}$ \\ ${ }^{1}$ Department of Obstetrics and Gynecology, Ruhr University Bochum, Marien Hospital Herne, D-44625 Herne; \\ ${ }^{2}$ Department of Senology, St. Anna Hospital, D-44649 Herne; ${ }^{3}$ Department of Pathology, \\ Hannover Medical School, Hannover, D-30625 Herne, Germany
}

Received March 9, 2015; Accepted March 18, 2016

DOI: $10.3892 / \mathrm{ol} .2016 .4514$

\begin{abstract}
Metastasis of ovarian cancer to the breast (MOCB) is a rare event. Clinical presentations of MOCB vary and surgery is the mainstay of treatment. The current study presents two cases of MOCB in women with recurrent ovarian cancer first diagnosed in April 2011 and October 2013, respectively. The patients presented to the clinic with a localized, palpable, painful mass in the upper outer quadrant of the right breast and a centrally localized, palpable, painful mass of the left breast, respectively. Breast sonography and mammography showed a singular, round, homogenous tumor with irregular borders in each case. An ipsilateral enlarged axillary node was palpable in one case. Tumor biopsy revealed an undifferentiated adenocarcinoma of unknown origin in one case and a moderately-differentiated adenocarcinoma suspected to be breast cancer in the other case. Tumor cells were positive for estrogen receptor and paired box 8 , and negative for GATA binding protein 3 in the two cases. Palliative mastectomy was performed in one case and lumpectomy with ipsilateral axillary sentinel node biopsy in the other case, and the final histology revealed MOCB in each. The post-operative course of the disease was uneventful and the patients continued with their ovarian cancer-specific chemotherapy. One patient succumbed to disease progression 2 months after breast surgery. The other patient remains alive and is currently undergoing systemic chemotherapy. The current study also presents a review of 110 cases of MOCB identified in a literature search of Pubmed. Data from these studies, including the clinical and histological characteristics of MOCB, and the clinical management and prognosis are discussed. Overall, MOCB is rare, with distinct clinical and histological features.
\end{abstract}

Correspondence to: Professor Clemens B. Tempfer, Department of Obstetrics and Gynecology, Ruhr University Bochum, Marien Hospital Herne, Hoelkeskampring 40, D-44625 Herne, Germany E-mail: clemens.tempfer@marienhospital-herne.de

Key words: ovarian cancer, intramammary metastasis, breast metastasis, non-mammary breast metastasis
The disease is usually treated with local surgical excision or mastectomy and has a poor prognosis.

\section{Introduction}

The typical course of ovarian metastasis is intraabdominal spread manifesting as peritoneal carcinomatosis. In addition, malignant pleural effusions are observed in $\sim 10 \%$ of women diagnosed with ovarian cancer (1). In contrast, metastatic lesions of the breast from an extramammary origin are rare events and account for $0.3 \%$ of malignant breast tumors (1). Metastatic lesions of the breast from an extramammary origin are rare events and account for $0.3 \%$ of malignant breast tumors (1). Metastatic lesions of the breast have a diverse appearance and tumor characteristics vary according to the site of the primary tumor. According to a Pubmed search performed in February 2015, using the search terms 'breast metastasis', 'ovarian cancer', 'non-mammary breast metastasis' and 'intramammary metastasis', 110 cases of metastasis of ovarian cancer to the breast (MOCB) have been reported in the literature. A study by Abbas et al described ultrasonographic and mammographic features in a series of 280 women with intramammary metastases, 41 of which were diagnosed with MOCB (2). In the study, intramammary masses and architectural distortion were the two main radiological patterns exhibited by the metastases. The masses also typically exhibited microlobulated margins and posterior enhancement on ultrasound. In a similar study, DeLair et al analyzed 85 cases of non-mammary metastases to the breast and axilla, 14 of which were MOCB (3). Notably, the ovaries was the predominant site of origin among all carcinomas causing metastases to the breast, comprising $58 \%$ of all cases. Morphologically, the majority of cases presented as a solitary nodule with noteworthy recurrent histological findings, including a metastatic lesion with a fibrous pseudocapsule and well-circumscribed growth pattern, and the lack of in situ carcinoma. The survival time was generally poor and $96 \%$ of patients succumbed to the disease, with a median overall survival time of 15 months post-diagnosis.

Karam et al investigated 29 ovarian cancer patients with malignant breast lesions, 10 of whom exhibited MOCB and 19 of whom presented with primary breast cancer (4). The study found marked differences between the two groups regarding 
disease-free survival time and the mean time interval between the diagnosis of ovarian cancer and the diagnosis of the breast tumor. Each time was significantly shorter for MOCB. The breast tumors of patients with MOCB were also less likely to be diagnosed on mammogram and the patients were less likely to have a family history of breast cancer. Overall survival time was significantly shorter for women with MOCB.

Another series of women with MOCB comprised 18 cases from a 14-year period (5). In this series, serous papillary carcinoma was the most common histological type of MOCB. Of the 18 patients, 4 presented with multiple breast lesions and 8 with a single metastasis, while 6 patients only exhibited involvement of the axillary lymph nodes. In 17/18 cases, the metastases exhibited papillary features, with psammoma bodies present in 4 cases. Immunoperoxidase studies showed positivity for Wilm's tumor (WT)-1 and negativity for prolactin-induced protein (GCDFP-15) in all cases.

Dursun et al reported 9 cases of bilateral MOCB (6). In this series, the mean survival time was 12 months. Smaller series of MOCB have also described 5 (7), 4 (1), 3 (8), 2 (9), 1 (10), 1 (11), 1 (12), and 1 (13) cases, respectively.

Histologically, the basis to the diagnosis of MOCB is that the papillary architecture consistent with serous papillary carcinoma is not a typical pattern of the majority of histological types of invasive breast carcinoma. Serous papillary carcinoma may resemble invasive micropapillary breast carcinoma and calcifications are observable in the two entities (1). In addition to histology, immunohistochemistry is frequently used to discern MOCB from other extramammary breast metastases and primary breast cancers. For example, the expression of WT-1, paired box 8 (PAX8) and mesothelin have been described as being useful in this regard (1-3).

The present study describes two cases of women with recurrent ovarian cancer and MOCB, and discusses the clinical and histological characteristics, and management of these patients.

\section{Case report}

Case 1. A 52-year-old woman, who was first diagnosed with recurrent ovarian cancer in April 2011, presented to the Department of Obstetrics and Gynecology, Ruhr University (Bochum, Germany) in June 2014 with a localized, palpable, painful mass in the upper outer quadrant of the right breast. The patient had no family history of breast cancer and no history of a previous breast pathology. Breast sonography and mammography revealed a singular, round, homogenous tumor with a well-defined border (Fig. 1). A singular, enlarged axillary lymph node was palpable. On mammography, the tumor presented as a loose, dense area with monomorphous calcifications, without clear borders (Fig. 1). The tumor was $0.5 \times 0.8 \mathrm{~cm}$ in size. A vacuum jet biopsy (Histocore ${ }^{\circledR}$ Automatic Biopsy system; BIP GmbH, Tuerkenfeld, Germany) was performed and the histology showed an undifferentiated adenocarcinoma of unknown origin. Immunohistochemically, the tumor cells expressed estrogen receptor (ER) and PAX8 and were negative for GATA binding protein 3 (GATA3) (Fig. 2). A lumpectomy was performed and the final histology confirmed a diagnosis of MOCB. The post-operative course of the disease was uneventful and the patient continued with ovarian cancer-specific chemotherapy, i.e., two cycles of
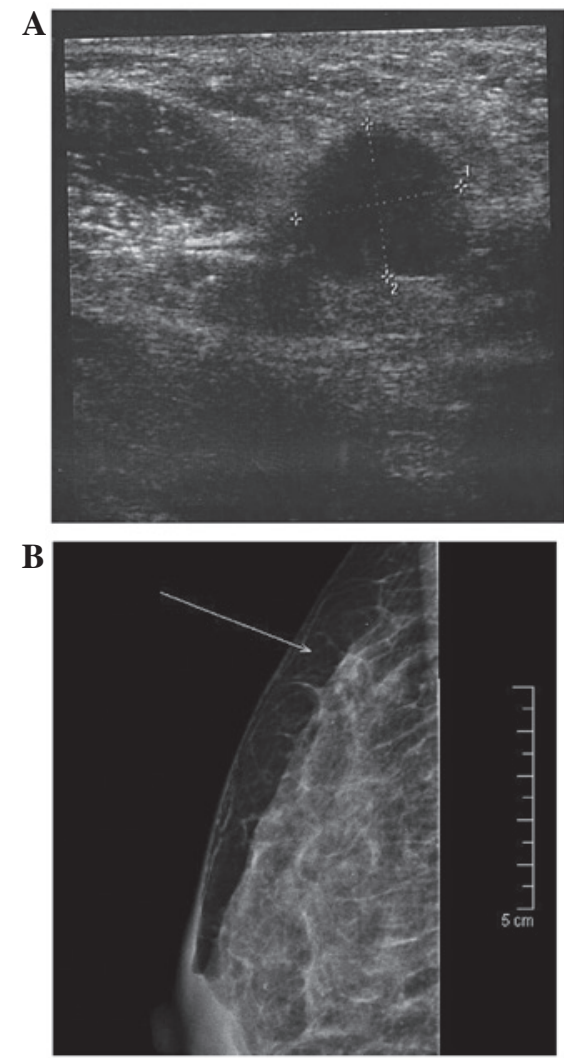

Figure 1. Case 1: (A) Ultrasonographic image demonstrating a singular, round, homogenous tumor and (B) a mammographic image demonstrating a loose, dense area (arrow) with monomorphous calcifications, without clear borders, suggestive of a malignant tumor.

topotecan $1.5 \mathrm{mg} / \mathrm{m}^{2}$ by intravenous infusion on days $1-5$ of a 21-day course. The patient succumbed to disease progression 2 months after breast surgery.

Case 2. A 51-year-old woman, who was first diagnosed with recurrent ovarian cancer in October 2013, presented to the Department of Obstetrics and Gynecology, Ruhr University in January 2014 with a centrally localized, palpable, painful mass in the left breast. The patient had no family history of breast cancer and no history of previous breast pathology. Breast sonography revealed a singular, lobulated, inhomogenous tumor with an irregular border. No enlarged axillary lymph nodes were palpable. On mammography, the tumor presented as a central, dense area without clear borders and with monomorphous, ring-shaped calcifications. The tumor was $8 \times 7.5 \mathrm{~cm}$ in size. A vacuum jet biopsy (Histocore Automatic Biopsy system; BIP GmbH) was performed and the histology showed a moderately-differentiated adenocarcinoma that was suspected of being a primary breast cancer. A mastectomy and ipsilateral axillary sentinel node resection were performed. The final histology revealed a diagnosis of MOCB and confirmed the ovarian origin of the lesion. Immunohistochemically, the tumor cells expressed ER and PAX8, and were negative for GATA3 (Fig. 3). The post-operative course of the disease was uneventful and the patient continued with ovarian cancer-specific chemotherapy, i.e., six cycles of liposomal doxorubicin $50 \mathrm{mg} / \mathrm{m}^{2}$ by intravenous infusion on day 1 of a 28-day course. After the 6 cycles, the patient was lost to follow-up. 


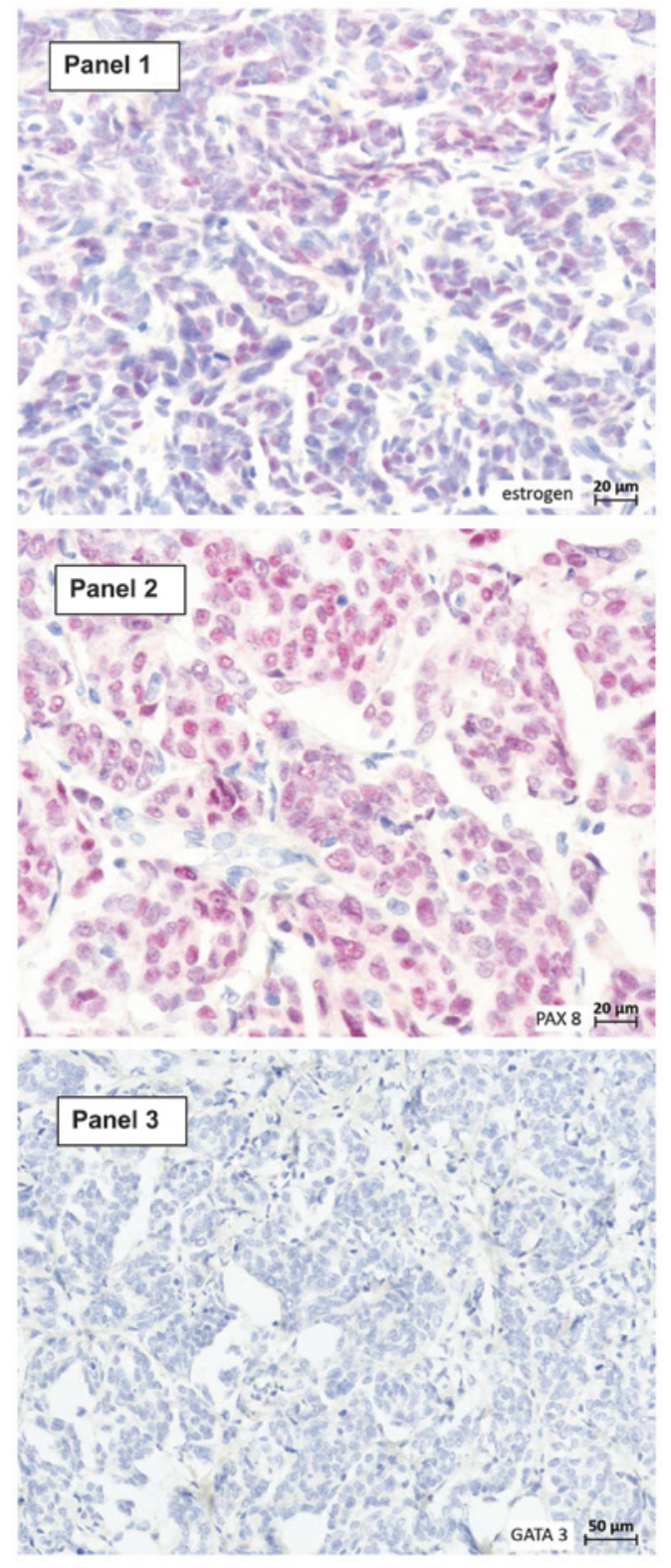

Figure 2. Case 1: Immunohistochemical specimens demonstrating the moderate expression of estrogen receptor (panel 1; magnification bar, $20 \mu \mathrm{m}$ ), the strong expression of paired box 8 (PAX8; panel 2; magnification bar, $20 \mu \mathrm{m}$ ) and the absence of expression of GATA binding protein 3 (GATA3; panel 3; magnification bar, $50 \mu \mathrm{m}$ ).

\section{Discussion}

The present study describes two cases of MOCB in women with recurrent ovarian cancer presenting as a solitary, painful breast mass. Breast sonography and mammography showed a singular, round, homogenous tumor with irregular borders in each case. Tumor biopsy revealed an undifferentiated adenocarcinoma of unknown origin in one case and a moderately-differentiated adenocarcinoma suspected of being breast cancer in the other case. The tumor cells were positive for ER and PAX8, and negative for GATA3 in the two cases. Lumpectomy was performed in one case and mastectomy with ipsilateral axillary sentinel node biopsy in the other case. The final histology revealed MOCB in each case. One patient succumbed to disease progression 2 months after

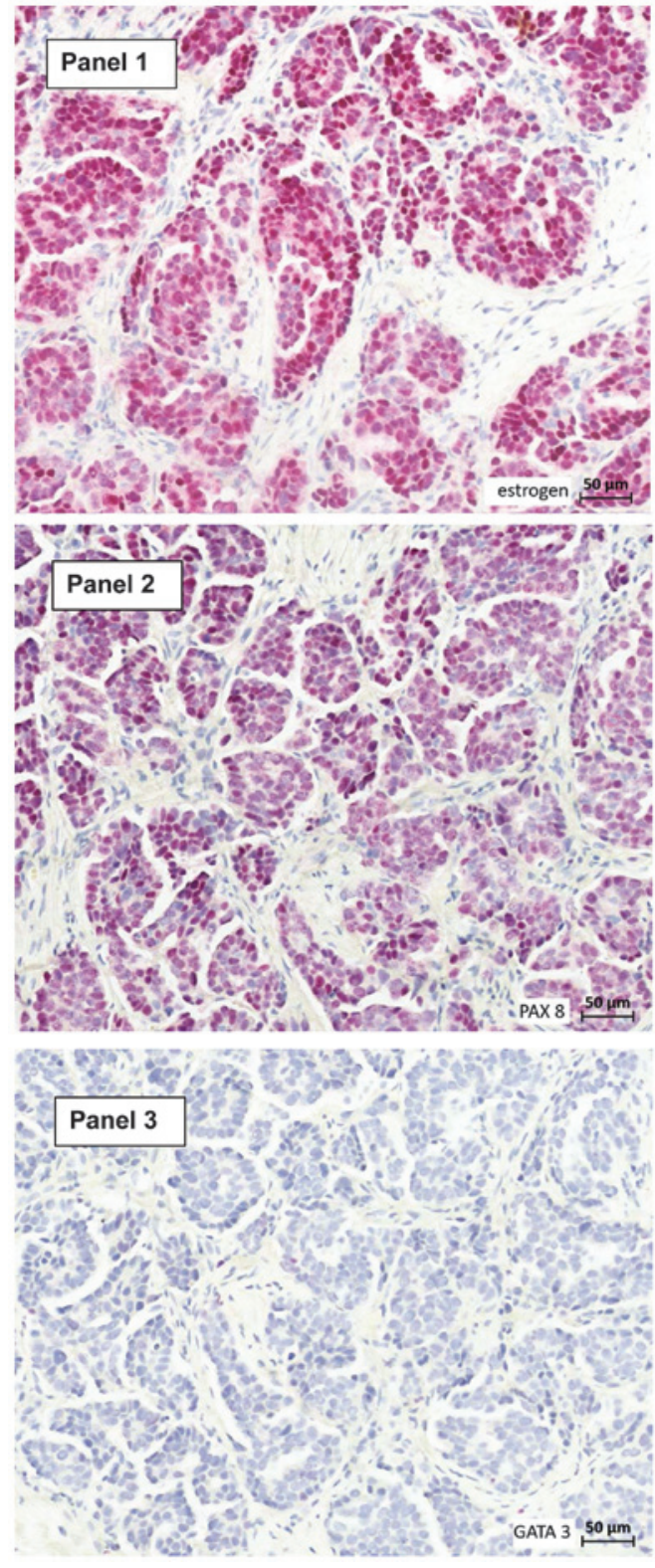

Figure 3. Case 2: Immunohistochemical specimens demonstrating the strong expression of estrogen receptor (panel 1; magnification bar, $50 \mu \mathrm{m}$ ), the strong expression of paired box 8 (PAX8; panel 2; magnification bar, $50 \mu \mathrm{m}$ ) and the absence of expression of GATA binding protein 3 (GATA3; panel 3; magnification bar, $50 \mu \mathrm{m})$.

breast surgery. The other patient remains alive and is currently undergoing systemic chemotherapy.

$\mathrm{MOCB}$ is a rare manifestation of recurrent ovarian cancer, with 110 cases reported in the literature (1-13). The typical morphological features of MOCB include a localized, painful mass with microlobulated margins and posterior enhancement on ultrasound (2) and a well-circumscribed growth pattern surrounded by a fibrous pseudocapsule, with notable absence of an in situ carcinoma (3). Local palliative surgical resection with free margins is the primary treatment of choice, and is consistently described in the literature (3-13). Although the local resection of the breast lesion does not positively affect the prognosis, it appears to be important to have an adequate specimen in order to establish the diagnosis of MOCB. This is difficult per se and even more so on a biopsy specimen only. 
Notably, in one of the present cases, the jet biopsy specimen was initially misdiagnosed as an undifferentiated adenocarcinoma of the breast. Bernadi et al (14) found that the status of resection margins and the management of infiltrated or narrow margins exerted no significant influence on local tumor recurrence rates or on overall patient survival. Instead, biological factors connected with tumor aggressiveness seem to play the most important role in breast cancer prognosis, independent of surgical radicality. The most important differential diagnoses of MOCB are primary breast cancer and extramammary metastases from a malignant tumor other than ovarian cancer. The unequivocal establishment of the diagnosis of MOCB is important, as primary breast cancer and extramammary metastases from a malignant tumor other than ovarian cancer require different therapies. The prognosis of MOCB has been described as poor, mostly reflecting the late stage of ovarian cancer progression (3-5). In one series, for example, $>90 \%$ of patients succumbed, with a median survival time of 15 months after diagnosis (3). This is consistent with the present study, in which one patient succumbed 2 months after the diagnosis of MOCB and the other patient was alive after a short follow-up of 3 months. In a series of 169 patients with confirmed metastases to the breast from non-breast solid organ primary tumors at the University of Texas M. D. Anderson Cancer Center, Williams et al (13) found that the median survival time from the diagnosis of breast metastasis was 10 months. On univariate analysis, a significantly higher survival rate was observed in patients who underwent surgical resection for breast metastases. On multivariate analysis, those individuals who did not undergo surgery were $88 \%$ more likely to succumb than those who underwent surgery (13). These data support the use of local surgery for the management of MOCB.

Histological classification of extramammary breast metastases and the differentiation from primary breast cancers is challenging, and is based on a combination of morphological and immunohistochemical features. For example, mammary and non-mucinous ovarian carcinomas are usually positive for cytokeratin 7, often positive for ER and typically negative for cytokeratin 20. The epithelial membrane antigen expression pattern is typical for serous papillary carcinoma, with expression on the outside of the papillary clusters and around the central spaces (1). The expression of WT-1 in the nuclei of cells occurs in $\sim 70 \%$ of ovarian carcinomas and in $95 \%$ of serous papillary carcinomas; however, $<10 \%$ of breast cancers exhibit this expression (15-18). GCDFP-15 expression is rarely observed in ovarian carcinoma $(1,19,20)$, whereas staining for cancer antigen (CA)125 is present in $~ 60 \%$ and $90 \%$ of ovarian and serous papillary carcinomas, respectively (1). CA125 expression is also normally observed in endocervical, endometrial, pancreatic and biliary carcinomas, but is less typically present in breast cancer $(1,21,22)$. Another immunohistochemical marker is mesothelin, which is expressed in $>90 \%$ of serous papillary carcinomas of the ovary, but is weakly expressed in 3-14\% of breast cancer cases (1,23-25). Lung, colorectal and gastric adenocarcinomas present with intermediate levels of staining.

The present study describes two cases of MOCB in women with recurrent ovarian cancer presenting as a solitary, painful breast mass. The lesions were positive for ER and PAX8, and negative for GATA3. Management consisted of local surgical resection without further treatment. In the literature, the prognosis of MOCB has been described as poor, which is attributed to the fact that MOCB represents a late stage of ovarian cancer progression. The histological diagnosis of MOCB is difficult and MOCB may be misdiagnosed as primary breast cancer. A number of immunohistochemical markers have been described as useful in the differential diagnosis of MOCB, including ER, PAX8, GATA3, CA125, WT-1, GCDFP-15 and mesothelin.

The case studies and literature review presented in this study add to the literature on MOCB characterizing this tumor as a late stage manifestation of ovarian cancer with a poor prognosis. Future clinical studies on MOCB should concentrate on conservative treatment and comprehensive histopathological diagnosis based on biopsy specimens with the goal of avoiding local surgery.'

\section{References}

1. Lee AH: The histological diagnosis of metastases to the breast from extramammary malignancies. J Clin Pathol 60: 1333-1341, 2007.

2. Abbas J, Wienke A, Spielmann RP, Bach AG and Surov A: Intramammary metastases: Comparison of mammographic and ultrasound features. Eur J Radiol 82: 1423-1430, 2013.

3. DeLair DF, Corben AD, Catalano JP, Vallejo CE, Brogi E and Tan LK: Non-mammary metastases to the breast and axilla: A study of 85 cases. Mod Pathol 26: 343-349, 2013.

4. Karam AK, Stempel M, Barakat RR, Morrow M and Gemignani ML: Patients with a history of epithelial ovarian cancer presenting with a breast and/or axillary mass. Gynecol Oncol 112: 490-495, 2009.

5. Recine MA, Deavers MT, Middleton LP, Silva EG and Malpica A: Serous carcinoma of the ovary and peritoneum with metastases to the breast and axillary lymph nodes: A potential pitfall. Am J Surg Pathol 28: 1646-1651, 2004.

6. Dursun P, Yanik FB, Kuscu E, Gultekin M and Ayhan A: Bilateral breast metastasis of ovarian carcinoma. Eur J Gynaecol Oncol 30: 9-12, 2009.

7. Zhou S, Yu B, Cheng Y, Xu X, Shui R, Bi R, Lu H, Tu X and Yang W: Metastases to the breast from non-mammary malignancies: A clinicopathologic study of 28 cases. Zhonghua Bing Li Xue Za Zhi 43: 231-235, 2014 (In Chinese).

8. Fulciniti F, Losito S, Botti G, Di Mattia D, La Mura A, Pisano C and Pignata S: Metastases to the breast: Role of fine needle cytology samples. Our experience with nine cases in 2 years. Ann Oncol 19: 682-687, 2008.

9. Amzerin M, Garcia C, Stanciu C, Veys I, Awada A, Errihani H and Gombos A: Case report: Mammary and rectal metastases from an ovarian cancer: Report of two cases and review of literature. F1000Res 3: 255, 2014.

10. Wood B, Sterrett G, Frost F and Swarbrick N: Diagnosis of extramammary malignancy metastatic to the breast by fine needle biopsy. Pathology 40: 345-351, 2008.

11. Yeh CN, Lin CH and Chen MF: Clinical and ultrasonographic characteristics of breast metastases from extramammary malignancies. Am Surg 70: 287-290, 2004.

12. Susini T, Olivieri S, Molino C, Castiglione F, Tavella K and Viligiardi R: Ovarian cancer initially presenting as intramammary metastases and mimicking a primary breast carcinoma: A case report and literature review. J Womens Health (Larchmt) 19: 169-174, 2010.

13. Williams SA, Ehlers RA II, Hunt KK, Yi M, Kuerer HM, Singletary SE, Ross MI, Feig BW, Symmans WF and Meric-Bernstam F: Metastases to the breast from nonbreast solid neoplasms: Presentation and determinants of survival. Cancer 110: 731-737, 2007.

14. Bernardi S, Bertozzi S, Londero AP, Gentile G, Angione V and Petri R: Influence of surgical margins on the outcome of breast cancer patients: A retrospective analysis. World J Surg 38: 22792287, 2014.

15. Cormio G, di Vagno G, Melilli GA, Loverro G, Cramarossa D and Selvaggi L: Ovarian carcinoma metastatic to the breast. Gynecol Obstet Invest 52: 73-74, 2001. 
16. Goldstein NS, Bassi D and Uzieblo A: WT1 is an integral component of an antibody panel to distinguish pancreaticobiliary and some ovarian epithelial neoplasms. Am J Clin Pathol 116 : 246-252, 2001

17. Lee BH, Hecht JL, Pinkus JL and Pinkus GS: WT1, estrogen receptor and progesterone receptor as markers for breast or ovarian primary sites in metastatic adenocarcinoma to body fluids. Am J Clin Pathol 117: 745-750, 2002.

18. Hwang H, Quenneville L, Yaziji H and Gown AM: Wilms tumor gene product: Sensitive and contextually specific marker of serous carcinomas of ovarian surface epithelial origin. Appl Immunohistochem Mol Morphol 12: 122-126, 2004.

19. Tornos C, Soslow R, Chen S, Akram M, Hummer AJ, Abu-Rustum N, Norton L and Tan LK: Expression of WT1, CA 125 and GCDFP 15 as useful markers in the differential diagnosis of primary ovarian carcinomas versus metastatic breast cancer to the ovary. Am J Surg Pathol 29: 1482-1489, 2005.

20. Kaufmann O, Deidesheimer $\mathrm{T}$ and Muehlenberg $\mathrm{M}$ : Immunohistochemical differentiation of metastatic breast carcinomas from metastatic adenocarcinomas of other common primary sites. Histopathology 29: 233-240, 1996.
21. Wick MR, Lillemoe TJ, Copland GT, Swanson PE, Manivel JC and Kiang DT: Gross cystic disease fluid protein-15 as a marker for breast cancer: Immunohistochemical analysis of 690 human neoplasms and comparison with alpha-lactalbumin. Hum Pathol 20: 281-287, 1989.

22. Lagendijk JH, Mullink H, van Diest PJ, Meijer GA and Meijer CJ: Immunohistochemical differentiation between primary adenocarcinomas of the ovary and ovarian metastases of colonic and breast origin. Comparison between a statistical and an intuitive approach. J Clin Pathol 52: 283-290, 1999.

23. Loy TS, Quesenberry JT and Sharp SC: Distribution of CA 125 in adenocarcinomas. An immunohistochemical study of 481 cases. Am J Clin Pathol 98: 175-179, 1992

24. Frierson HF, Moskaluk CA, Powell SM, Zhang H, Cerilli LA, Stoler MH, Cathro H and Hampton GM: Large scale molecular and tissue microarray analysis of mesothelin expression in common human carcinomas. Hum Pathol 34: 605-609, 2003.

25. Ordonez NG: Application of mesothelin immunostaining in tumor diagnosis. Am J Surg Pathol 27: 1418-1428, 2003. 